\title{
Refinement of Orthogonal Graph Drawings *
}

\author{
Janet M. Six,, Konstantinos G. Kakoulis, and Ioannis G. Tollis \\ CAD \& Visualization Lab \\ Department of Computer Science \\ The University of Texas at Dallas \\ P.O. Box 830688, EC 31 \\ Richardson, TX 75083-0688 \\ \{janet,kostant, tollis\}@utdallas.edu
}

\begin{abstract}
Current orthogonal graph drawing algorithms produce drawings which are generally good. However, many times the readability of orthogonal drawings can be significantly improved with a postprocessing technique, called refinement, which improves aesthetic qualities of a drawing such as area, bends, crossings, and total edge length. Refinement is separate from layout and works by analyzing and then fine-tuning the existing drawing in an efficient manner. In this paper we define the problem and goals of orthogonal drawing refinement and introduce a methodology which efficiently refines any orthogonal graph drawing. We have implemented our technique in $\mathrm{C}++$ and conducted preliminary experiments over a set of drawings from five well known orthogonal drawing systems. Experimental analysis shows our technique to produce an average $34 \%$ improvement in area, $22 \%$ in bends, $19 \%$ in crossings, and $34 \%$ in total edge length.
\end{abstract}

\section{Introduction}

Orthogonal graph drawings represent nodes with boxes and edges with polygonal chains of horizontal and vertical line segments which reside on an underlying grid. Drawings in this style are useful for applications which benefit from high clarity representations. Much research has been conducted in this area and various algorithms exist to produce orthogonal drawings of planar 1 111 162729 , general maximum degree four [1,23,26], and general higher degree graphs [2,14,21]. An extensive experimental study was conducted by Di Battista et. al. 7] where four general purpose orthogonal drawing algorithms were implemented and compared with respect to area, bends, crossings, edge length, and running time.

Many papers have suggested ways of evaluating the "goodness" of a graph drawing (for example $8,10,17 / 20,28$ ) in addition to the standard measures of area, crossings, bends, and edge length which are used as a means to evaluate the quality of a graph drawing algorithm. The achievement of many of these goals,

\footnotetext{
* Research supported in part by NIST, Advanced Technology Program grant number 70NANB5H1162 and by the Texas Advanced Research Program under Grant No. 009741-040.
} 
aesthetics and constraints is known to be NP-Hard. Complicating this issue is the experience that maximizing one particular quality of a drawing causes another to be significantly poor since some of these qualities work against each other. Therefore most algorithms try to layout the graph in a manner which is good for some set of aesthetics.

Current orthogonal graph drawing algorithms produce drawings which are generally good. However, many times the readability of orthogonal drawings can be significantly improved with a postprocessing step which modifies the positions of nodes, edges, and bends. It is vital that drawings which are created by any graph layout system are very readable. Refinement is a postprocessing methodology which can be applied to any orthogonal drawing and improves readability by analyzing the drawing and then fine-tuning it while keeping the majority of the layout intact. The result is a new drawing which has improved aesthetic qualities including area, bends, crossings, and edge length. Previous work includes compaction strategies $[2,27,29]$ and movement of stranded nodes [12]. However, the scope of these postprocessing techniques is limited. A more sophisticated methodology is needed to further improve the aesthetic qualities of graph drawings. We have focused on the development and implementation of several efficient refinement modules which work on any orthogonal drawing (including degree greater than four). An example of a drawing before and after refinement is shown in Figure 1.
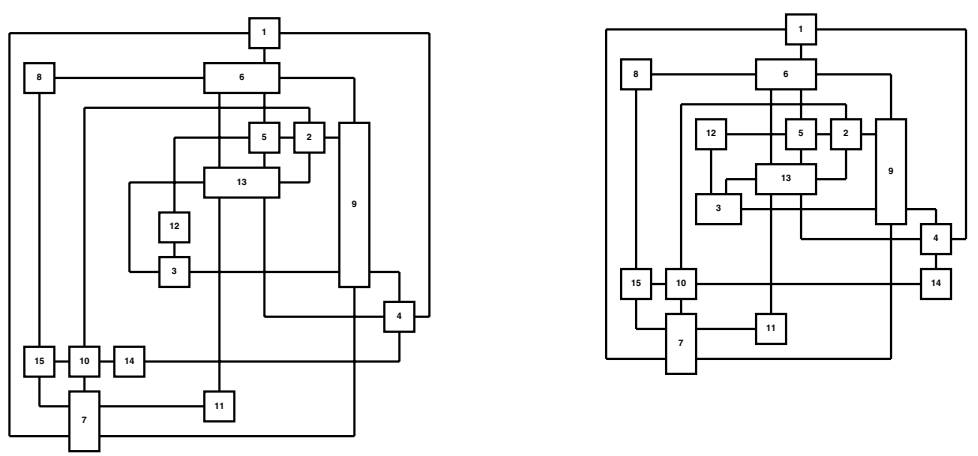

Fig. 1. One of the Rome graphs as drawn with GIOTTO on the left. The same drawing after refinement on the right (same scale). There is a $29 \%$ improvement in area, $13 \%$ improvement in the number of bends, $11 \%$ in the number of crossings, and $24 \%$ in the total edge length.

There are two types of refinement: interactive and static. During the interactive refinement of drawings we must maintain the user's mental map [20] and are allowed to make only minimal changes. The requirements for the refinement methodology are very similar to those of interactive graph drawing algorithms 


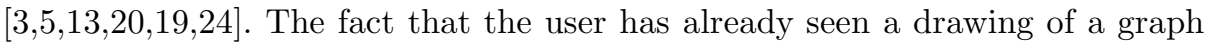
means that the refinement technique must not make changes so drastic that pieces of the drawing are not recognizable. Static refinement fine tunes drawings for which we do not have to maintain the user's mental map: we are free to make any change in the embedding. Certainly refinement cannot be a cure for a very poor layout because this would require the essential invocation of some other layout algorithm. Refinement fine-tunes an existing drawing by improving some layout qualities. Our tool performs static refinement on any orthogonal graph drawing.

Our refinement technique produced a significant $19 \%$ to $34 \%$ average improvement for each of the generally accepted characteristics area, bends, crossings, and total edge length in preliminary experiments over drawings from five algorithms. Since different applications require different classes of drawings and therefore need to focus on varying kinds of refinement, our system has the flexibility to vary the types and order of refinement modules called, so that a user may refine drawings in a manner specific for a particular application.

\section{Refinement}

During a survey of orthogonal drawings from a variety of sources, we repeatedly observed extra area, bends, edge crossings, and edge length caused by U-turns in edges (as described in [19]), superfluous bends, poor placement of degree two nodes, two incident edges of a node crossing, nodes stranded very far from their neighbors, and unused space inside the drawing. See Figure 2 for examples. Note that the attachment points of the left three vertical edges in the self crossing example, Figure 2D, are not moved. The node is extended to the left allowing the placement of the edge with the bend. Then the right side of the node is contracted since the space of the old edge placement is no longer needed.

Specifically, U-Turns are three contiguous edge sections which form a "U" shape with the middle section pulled far from the source and target nodes of those three sections. Superfluous bends are those which exist even if there is room in the space of the drawing for an edge with less bends. Clearly U-Turns and superfluous bends can occur multiple times in edges which have four or more sections. Poorly placed degree two nodes are those which are neither on a bend nor in the midst of its two incident edges. Self crossings are those which occur between two edges incident to the same node. Self crossings are divided into two categories: near and far self crossings. Near self crossings are those whose positions differ from that of the node in either the horizontal or vertical orientation. Far self crossings differ in both orientations. A stranded node is a degree one node which is placed very far from its neighbor.

Fixing a set of the above defined problems with a sequence of refinements will certainly reduce the visual complexity of the drawings, however we take our methodology one step further. If we perform these refinements directly on the given drawing we will improve the quality of the drawing, but will still miss some of those improvements which are less visually obvious. Therefore we preprocess 

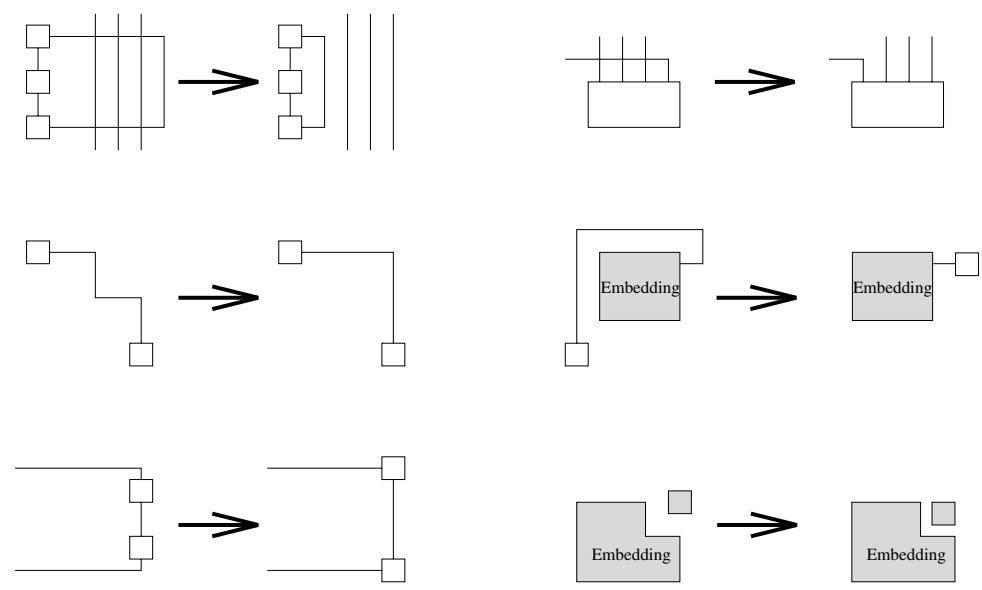

Fig. 2. Examples of the problems we solve with a refinement technique: A: Uturns, B: superfluous bends, C: poor placement of degree two nodes, D: selfcrossings, E: stranded nodes, and F: extra area.

the given graph into a simpler one. First we absorb all chains of degree two nodes into an edge and then denote each degree one node to be a super node and determine the minimum distance needed between it and its neighbor (as is also done in [521]). All refinement operations are performed on this simplified graph. The refinement techniques have been implemented to acknowledge the presence of super nodes and edges and place them in some appropriate manner. After refinement is complete the preprocessing is undone in order to restore the graph to its original topology. The preprocessing operations allow our methodology to catch significantly more of the above problems and therefore produce better quality drawings.

Procedure: Refine-Orthogonal-Graph-Drawing

Input: An orthogonal graph drawing, $\Gamma$, of a graph, $G$

Output: A new orthogonal drawing, $\Gamma^{\prime}$, of $G$ with a lower visual complexity

1. Build the abstracted graph, $G^{\prime}$, of $G$, such that

(a) Each chain of degree two nodes is abstracted into a single edge.

(b) Each degree one node is denoted to be a super node and the minimum necessary distance between it and its lone neighbor is calculated. The minimum distance is directly proportional to the number of absorbed degree two nodes in the lone incident edge.

2. For each edge, $e$, in $G^{\prime}$

(a) If $e$ contains a sequence of three edge segments which form a U-Turn edge, then pull in the middle segment of that sequence so that it is as close as possible to its source and target.

(b) If $e$ contains a sequence of three edge segments which have an extra bend and there exists room for a lower bend edge routing in the drawing 
then replace the current routing with the lower bend solution. Use the bend-stretching transformations of [29].

(c) If $e$ is an edge which represents a chain of degree two nodes in $G$, postprocess this edge to restore the degree two nodes and verify that each degree two node is either on a bend or in the midst of its two incident edges.

3. For each node, $v$, in $G^{\prime}$

(a) If $v$ has a near self crossing, expand the node by one row or column in the appropriate direction and move the attachment point of the trouble edge to that new row or column. Place any abstracted degree two nodes so that they do not occlude any other drawing element. If $v$ has a far self crossing and is degree two, then place the node at the location of the crossing. Otherwise add one row or column and break the crossing into a knock-knee [18] edge routing. Both of these far self crossing solutions swap the attachment points of the crossing edges at $v$ so that neither of the neighbor nodes is moved.

(b) If $v$ is a stranded node, place it as close to the neighbor node as space allows in $\Gamma$.

4. Perform a VLSI layout compaction [915]18] to remove extra space in the drawing.

5. Undo the preprocessing of step 1.

Many improvements may be made without increasing the area of the drawing, but allowing the addition of more area may enable refinement to significantly improve other aesthetics of the drawing. For example, adding a row or column may be necessary to remove a self crossing. However this allowance should be according to user requirements and must be parametrically defined.

It is important to emphasize that refinement is an evolving process. We are planning to implement additional modules for improving orthogonal drawings as we discover and develop further techniques.

\section{Implementation and Experimental Results}

\subsection{Implementation}

Refine-Orthogonal-Graph-Drawing has been implemented in GNU C++ Version 2.7.2 with Tom Sawyer Software's Graph Layout Toolkit Version 2.3.1 and a TCL/TK graphical user interface. A set of experiments has been run on a Sparc 5 running Sun OS Release 4.1.3.

Many interesting and challenging issues were addressed during the implementation of Refine-Orthogonal-Graph-Drawing. First we needed a mechanism to search the space within the given drawing to move pieces of the drawing without occluding uninvolved elements. We represent the space of the drawing with a dynamic orthogonal grid structure in which rows and columns may be added at any point within the space. Elements of the drawing are represented with grid segments owned by nodes, edges, and bends. 
Each of the refinement modules can be viewed as a local searching technique. The module which shortens U-Turn edges looks at the endpoints of the middle edge and places them as close as possible to their neighbors. If the new placement of the middle segment is still orthogonal and does not occlude any drawing elements we are done. Otherwise we search the space toward the old placement until sufficient space is found for the middle segment. At worst, this will be the old placement. See Figure 3 It is important to note that the edges involved in the U-Turn may actually represent a chain of degree two nodes, therefore we must detect that situation and be sure to place those nodes only where there is sufficient space in the grid. We iteratively place each degree two node closer to its neighbor. Also, we examine each set of three contiguous segments in each edge so that we can catch more of the U-Turns. This is especially important when we are really dealing with degree two chains.
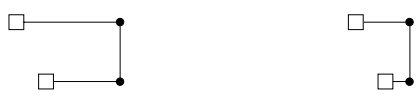

Fig. 3. Fixing a U-Turn edge. The left illustration shows the involved elements in their original positions. The endpoints of the middle segment are shown with circles and the source and target of the U-Turn with boxes. The right illustration shows the final placement of the middle edge.

The superfluous bends module examines each set of three contiguous edge segments in every edge. For each set, call one endpoint of the middle edge segment $x$ and the other $y$. Define $a$ and $b$ to be the points shown in Figure 4. If space in the drawing allows, then place $x$ at $a$ or $y$ at $b$.

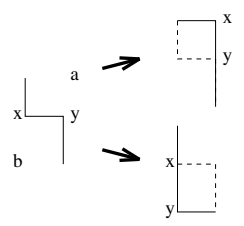

Fig. 4. Superfluous bend solutions. The old edge routing is shown with a dashed line in the two illustrations on the right.

The self crossing module inserts rows or columns as specified in Step 3 of Procedure Refine-Orthogonal-Graph-Drawing. Since the grid is dynamic, we insert the new gridlines inside the node to fix a near self crossing on the appropriate side and that automatically forces the node to grow. For far self crossings of a degree three or higher node, a row or column is inserted at that crossing (see 
Figure 5 for examples). The first far self crossing solution is for the case where the node has degree two while the second solution is for higher degree nodes. The second solution adds two bends and therefore the superfluous bends refinement module should be run on the new drawing to remove these extra bends if possible. Some users may not wish to save a crossing at the potential cost of a new row and two additional bends, therefore the user is given a parametric option whether or not to use this particular solution. Also note that in a far crossing the attachment points of the two crossing edges are swapped so that the positions of those neighbors are not changed. If the movement of the attachment points is not acceptable, the user may set a parametric option preventing this action.

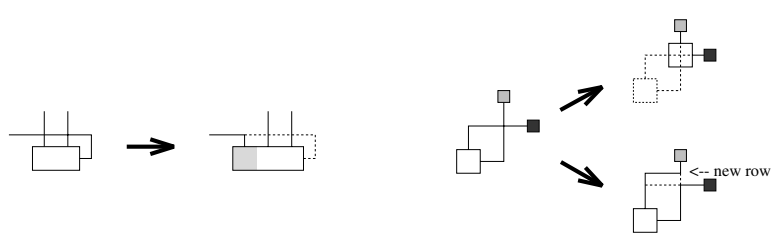

Fig. 5. Near and far self crossing solutions. Old node placements and edge routings are shown with dashed lines.

The stranded node module removes involved node and edge segments from the grid and searches the space from the neighbor node out. If the lone incident edge actually represents a chain of degree two nodes, this process is iteratively carried out for each degree two node from the original node out to the stranded node, see Figure 6.

The user also has a parametric option for this module to allow the addition of rows and columns as necessary to avoid adding any crossings or to allow crossings to remain. This option gives the user the ability to decide to give priority to area or crossing reduction. We believe that reducing the number of crossings is paramount and chose the first parametric option for our experiments. In part, this decision was influenced by the study presented in [25] which showed the number of crossings to have a very significant effect on readability. This is certainly not to say that avoiding crossings will always cause the addition of area. In fact, in many drawings, the area will still be reduced while avoiding crossings.

The module which fixes poor placement of degree two nodes, first postprocesses edges which represent degree two chains and then visits each newly restored degree two node to verify that it lies either on a bend or in the midst of its two incident edges.

The implementation of the compaction module is inspired by one dimensional VLSI layout compaction 91518. A one dimensional graph-based compaction is performed once each for the horizontal and vertical directions. Similar types of compaction modules have been used in [2 27,29]. 

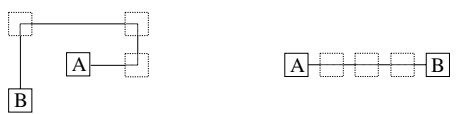

Fig. 6. Fixing a stranded node with an edge that represents a degree two chain. Notice that not only are we placing related nodes closer to each other, but also unwrapping a poorly routed edge.

It is recognized that different users may want different types of drawings and need to refine a specific aesthetic quality. Also different algorithms merit the use of different types of refinement: drawing algorithms inherently have strengths and weaknesses with respect to different aesthetic criteria. Static orthogonal graph drawing algorithms either planarize and then embed with a planar algorithm or proceed in an essentially incremental fashion. While the problems described in the previous section occur with all of the orthogonal algorithms surveyed, some types of problems occur more frequently in a particular class of orthogonal algorithms. For example, planarization algorithms have a tendency to have some very long edges and place nodes far away from their neighbors. Incremental algorithms tend to have superfluous bends. Our refinement methodology automatically detects these problems and fixes them regardless of the class of algorithm used to create the drawing. Furthermore, our implementation communicates with the user via a graphical user interface which allows the user to perform the desired set of refinements in any order. This flexibility adds power to refinement in that the user can refine any orthogonal drawing in a manner which is application specific.

We have designed and implemented all of our refinement techniques to take linear time with respect to the number of grid segments. The number of grid segments is bounded by the number of nodes and edges and hence refinement takes $O(n+m)$ time, where $n$ is the number of nodes and $m$ is the number of edges.

\subsection{Experimental Results}

We have conducted a set of preliminary experiments with our implementation. The source drawings are layouts of Rome graphs [7] (available at http://www . inf . uniroma3.it/people/gdb/wp12/LOG.html) produced by the Giotto, BendStretch, Column, and Pair algorithms as implemented at Brown University's Graph Drawing Server 4] (http://loki.cs.brown.edu:8081/graphserver), and Tom Sawyer Software's Graph Layout Toolkit version 2.3.1. Each drawing was given as input to our refinement implementation and data collected as to the improvements made in area, bends, edge crossings, and total edge length for each drawing. A table summarizing the average percent improvement for this set of aesthetics over drawings from the five layout algorithms is given next. 


\begin{tabular}{|c|c|c|c|c|c|c|c|c|c|c|c|c|}
\hline & \multicolumn{2}{|c|}{ BEND-ST } & \multicolumn{2}{|c|}{\begin{tabular}{||c|c|} 
COLUMN \\
\end{tabular}} & \multicolumn{2}{|c|}{ GIOTTO } & \multicolumn{2}{|c|}{$\overline{\mathrm{PAIR}}$} & \multicolumn{2}{|c|}{ GLT } & \multicolumn{2}{|r|}{$\Delta$} \\
\hline & All & Better & All & Better & All & Better & All & 3etter & All & Better & All & Better \\
\hline Area & 20 & 27 & 52 & 53 & 11 & 17 & 55 & 55 & 32 & 39 & 34 & 38 \\
\hline Bends & 20 & 27 & 40 & 40 & 2 & 8 & 37 & 37 & 11 & 21 & 22 & 27 \\
\hline Crossings & 9 & 40 & 41 & 43 & 1 & 9 & 24 & 29 & 20 & 27 & 19 & 30 \\
\hline \begin{tabular}{|l|} 
Edge \\
Length \\
\end{tabular} & 30 & 32 & 51 & 51 & 21 & 22 & 45 & 45 & 21 & 28 & 34 & 36 \\
\hline$\delta$ & 20 & 32 & 46 & 47 & 9 & 14 & 40 & 42 & 21 & 29 & & \\
\hline
\end{tabular}

In the table, the left column of percentages for each algorithm represents the average improvement over all drawings. This includes the drawings for which our technique is unable to improve the drawing with respect to that aesthetic. The second column of percentages for each layout technique represents the average percent improvement for those drawings which our technique was able to strictly improve. The row marked $\delta$, represents the average percent improvement made with respect to all four aesthetics for that particular algorithm. $\Delta$ represents the average percent improvement made for area, bends, crossings or edge length over all of the experimental source drawings. Note that refinement acts on the input drawing which is produced by a specific implementation of a chosen algorithm. As such, refinement improves aesthetic qualities caused by possible problems of both a chosen algorithm and the implementation of that algorithm.

Our implementation of refinement makes a $34 \%$ improvement on average in both area and total edge length. This huge improvement is due largely to the modules of our technique which shorten long edges. As it is well known in the VLSI circuit design field, the area is usually dominated by the amount of wiring. Likewise, the area of graph drawings is usually dominated by the edge lengths. So our methods which shorten the total edge length also inherently decrease the area. Hence we see a proportional improvement in both area and total edge length. In addition the area is also reduced by the compaction phase. Although several orthogonal layout algorithms already use a compaction phase, our compaction has such a significant effect since other phases of refinement have simplified the drawing by reducing its geometric complexity. Therefore, pieces of the drawing have more freedom to move and can therefore be compacted more efficiently.

Our experiments also show about $20 \%$ improvement on average with respect to bends and crossings. This is due to the modules which particularly refine those elements.

Refinement significantly improves drawings created by each of these orthogonal algorithms. As mentioned earlier, every layout algorithm has a set of strengths and weaknesses. These strengths and weaknesses with respect to area, bends, crossings and total edge length are apparent in the numbers collected for each algorithm in our experiments. Giotto is the most evolved implementation of the Graph Drawing Server (GDS) and thus refinement has a lesser impact on these drawings. One of the main steps of Giotto finds the minimum number of bends of the planarized graph [29]. Our refinement improved the number of bends by an average $2 \%$. When our tool was able to improve the number of bends, the 
improvement was on the average $8 \%$, with some improvements up to $50 \%$. The planarization step of Giotto causes some nodes to be placed far from their neighbors, hence we see a more significant improvement of $21 \%$ with respect to total edge length.

Likewise we notice similar behavior with Tom Sawyer Software's Graph Layout Toolkit (GLT). Their implementation allows each edge to have at most one bend. So the average is $11 \%$ compared to the average $22 \%$ improvement in the number of bends over the entire experiment set. Column and Pair drawings experience very significant improvements of each aesthetic. Especially notice the average $40 \%$ and $37 \%$ improvements in the number of bends and the average $51 \%$ and $45 \%$ improvements in total edge length. This is related not only to the nature of these algorithms, but also to the implementation of these algorithms in the GDS. As discussed above, the quality of a drawing before refinement depends heavily on a chosen algorithm, and even more heavily on the implementation of the algorithm. Refinement fixes problems caused by both the algorithms and their implementations. This is evidenced by the behavior of refinement on Column and Pair drawings. Instead of dividing input graphs into biconnected components and performing a layout on each component, the GDS implementations of Column and Pair augment graphs to make them biconnected and then perform the layout on the augmented graph. The augmenting edges are removed during the final step and the resulting drawing shows only the input graph. This implementation decision has increased the geometric complexity of many Column and Pair drawings. Our refinement technique provides a 9\% to 30\% average improvement of Bend-Stretch drawings for all aesthetics considered.

It is important to note the difference between the "All" percentages and the "Better" percentages. All five of these orthogonal algorithms produce good drawings: sometimes the number of crossings and bends is already optimal. Of course, the refinement tool cannot reduce the number of bends in a drawing which already has the lowest possible number of bends. Also, some layouts do not allow the embedding to be refined much. Hence, the "All" percentage improvement is lower than the strictly "Better" improvement.

Example drawings from Bend-Stretch, Column, Pair and Tom Sawyer's GLT along with their refined drawings are included in the following pages. Such an example for Giotto appears in Figure 1

\section{Conclusions and Future Work}

In this paper we presented an efficient postprocessing technique to improve aesthetic qualities of any orthogonal graph drawing. Specifically we focused on reducing the area, number of bends, number of crossings and total edge length. A preliminary experimental study conducted over a set of drawings from five well known algorithms produced very good results. An average 34\% improvement was made in area, $22 \%$ in bends, $19 \%$ in crossings, and $34 \%$ in edge length.

Refinement is an evolving technique. We plan to implement more modules of refinement as we develop further techniques to improve orthogonal graph 

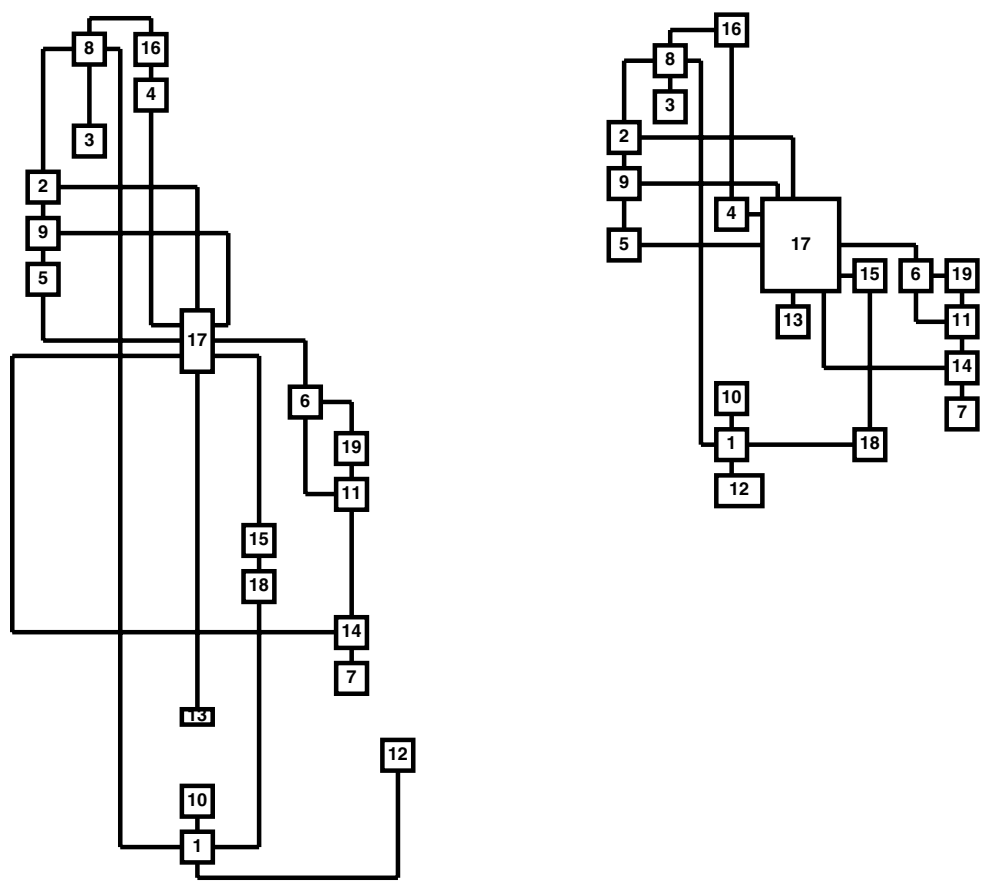

Fig. 7. One of the Rome graphs as drawn with Column on the left. The same drawing after refinement on the right (same scale). There is a $47 \%$ improvement in area, $42 \%$ improvement in the number of bends, $40 \%$ in the number of crossings, and $56 \%$ in the total edge length.

drawings. Also, we plan to further enhance the graphical user interface of our refinement tool so that the refinement process can be even more tailored to an individual user's needs. More parametric options, such as the one for the stranded node module which sets a priority for crossings or area, will be added. Further, we would like to capture the user's modal interactions with the drawing elements so that we can further improve the quality of given drawings to some application-specific standard.

\section{Acknowledgments}

The authors would like to thank Uğur Doğrusöz and Therese Biedl for their technical advice and Tom Sawyer Software, especially its President and CEO Brendan Madden, for making their software available to implement our refinement modules. We are also grateful to Stina Bridgeman, Roberto Tamassia and the Graph Drawing group at Brown University for the Graph Drawing Server which is a great resource. 

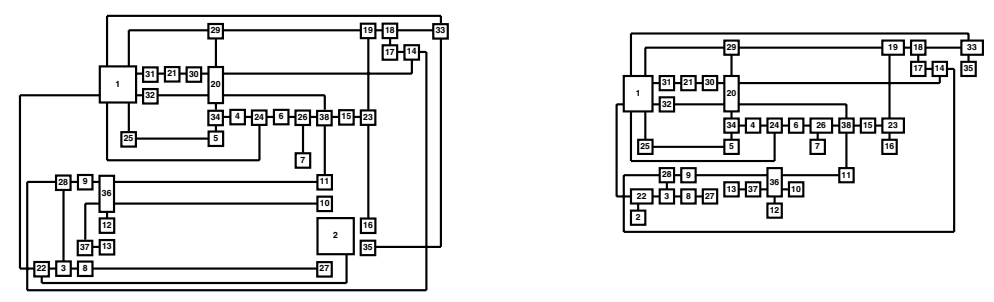

Fig. 8. One of the Rome graphs as drawn with Bend-Stretch on the left. The same drawing after refinement on the right (same scale). There is a $34 \%$ improvement in area, $24 \%$ in the number of bends, $33 \%$ in the number of crossings, and $39 \%$ in the total edge length.
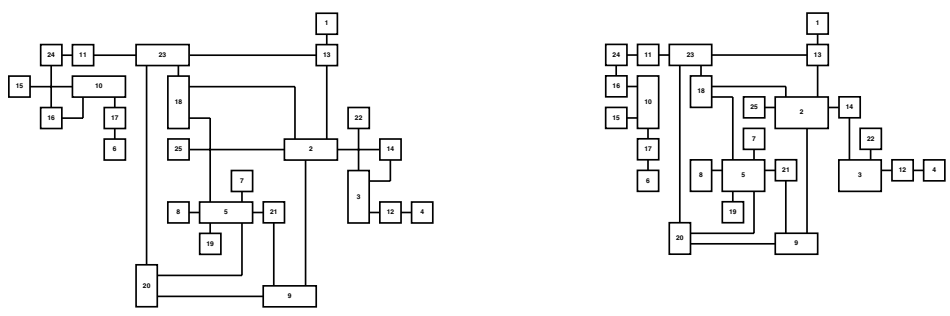

Fig. 9. One of the Rome graphs as drawn with Tom Sawyer Software's GLT 2.3.1 on the left. The same drawing after refinement on the right (same scale). There is a $34 \%$ improvement in area, $40 \%$ improvement in the number of bends, $100 \%$ in the number of crossings, and $36 \%$ in the total edge length.

\section{References}

1. T. Biedl and G. Kant, A Better Heuristic for Orthogonal Graph Drawings, Proc. ESA '94, LNCS 855, Springer-Verlag, 1994, pp. 24-35.

2. T. C. Biedl, B. P. Madden and I. G. Tollis, The Three-Phase Method: A Unified Approach to Orthogonal Graph Drawing, Proc. GD '97, LNCS 1353, SpringerVerlag, 1997, pp. 391-402.

3. S. Bridgeman, J. Fanto, A. Garg, R. Tamassia and L. Vismara, Interactive Giotto: An Algorithm for Interactive Orthogonal Graph Drawing, Proc. GD '97, LNCS 1353, Springer-Verlag, 1997, pp. 303-308.

4. S. Bridgeman, A. Garg and R. Tamassia, A Graph Drawing and Translation Service on the WWW, Proc. GD '96, LNCS 1190, Springer-Verlag, 1997, pp. 45-52.

5. R. F. Cohen, G. Di Battista, R. Tamassia and I. G. Tollis, Dynamic Graph Drawings: Trees, Series-Parallel Digraphs, and Planar ST-Digraphs, SIAM J. Computing, 24(5), October 1995, pp. 970-1001. 

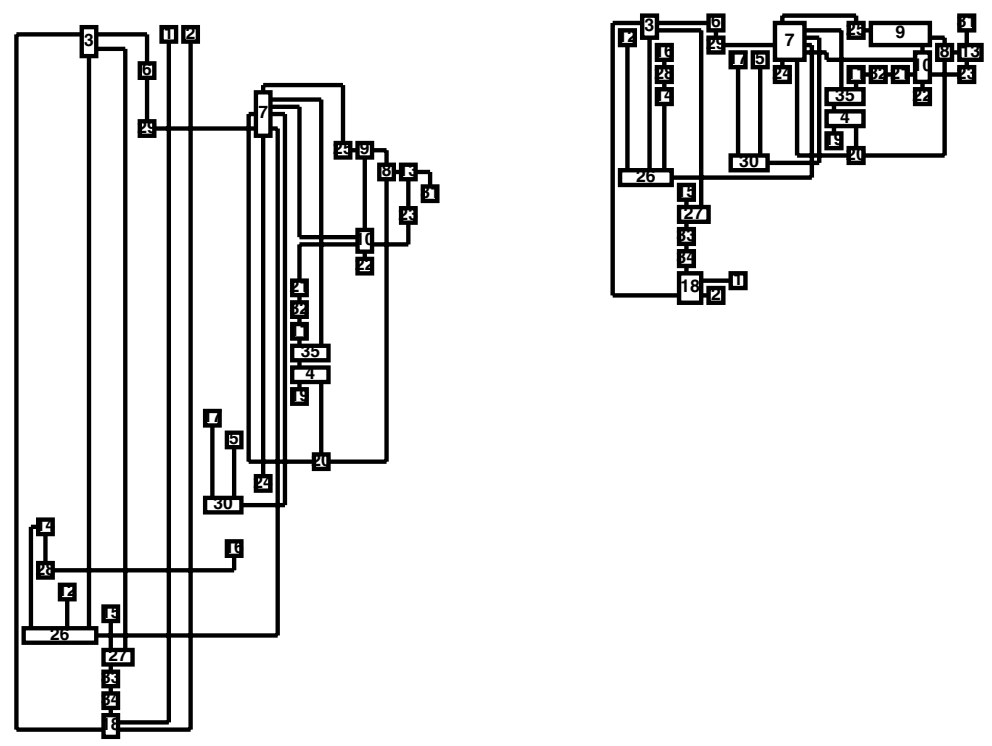

Fig. 10. One of the Rome graphs as drawn with PAIR on the left. The same drawing after refinement on the right (same scale). There is a $65 \%$ improvement in area, $35 \%$ improvement in the number of bends, $56 \%$ in the number of crossings, and $66 \%$ in the total edge length.

6. G. Di Battista, P. Eades, R. Tamassia and I. G. Tollis, Algorithms for Drawing Graphs: An Annotated Bibliography, Computational Geometry: Theory and Applications, 4(5), 1994, pp. 235-282.

7. G. Di Battista, A. Garg, G. Liotta, R. Tamassia, E. Tassinari and F. Vargiu, An Experimental Comparison of Four Graph Drawing Algorithms, Computational Geometry: Theory and Applications, 1997, pp. 303-325.

8. C. Ding and P. Mateti, A Framework for the Automated Drawing of Data Structure Diagrams, IEEE Transactions on Software Engineering, 16(5), 1990, pp. 543-557.

9. J. Doenhardt and T. Lengauer, Algorithmic Aspects of One Dimensional Layout Compaction, IEEE Transactions on Computer-Aided Design of Integrated Circuits and Systems, 6(5), 1987, pp. 863-879.

10. C. Esposito, Graph Graphics: Theory and Practice, Computers and Mathematics with Applications, 15(4), 1988, pp. 247-253.

11. S. Even and G. Granot, Rectilinear Planar Drawings with Few Bends in Each Edge, Tech. Report 797, CS Dept., Technion, Israel Inst. of Tech., 1994.

12. Jody Fanto, Postprocessing of GIOTTO drawings, http://www.cs.brown.edu/ people/jrf/.

13. U. Fößmeier, Interactive Orthogonal Graph Drawing: Algorithms and Bounds, Proc. GD '97, LNCS 1353, Springer-Verlag, 1997, pp. 111-123.

14. U. Fößmeier and M. Kaufmann, Algorithms and Area Bounds for Nonplanar Orthogonal Drawings, Proc. GD '97, LNCS 1353, Springer-Verlag, 1997, pp. 134-145. 
15. M. Y. Hsueh, Symbolic Layout and Compaction of Integrated Circuits, Ph.D. Thesis, University of California at Berkeley, Berkeley, CA, 1979.

16. G. Kant, Drawing Planar Graphs Using the lmc-ordering, Proc. 33rd Ann. IEEE Symposium on Found. of Comp. Sci., 1992, pp. 101-110.

17. C. Kosak, J. Marks and S. Shieber, Automating the Layout of Network Diagrams with Specified Visual Organization, IEEE Transactions on Systems, Man, Cybernetics, 24(3), 1994, pp. 440-454

18. Thomas Lengauer, Combinatorial Algorithms for Integrated Circuit Layout, John Wiley and Sons, 1990.

19. K. Miriyala, S. W. Hornick and R. Tamassia, An Incremental Approach to Aesthetic Graph Layout, Proc. Int. Workshop on Computer-Aided Software Engineering (Case '93), 1993, pp. 297-308.

20. K. Misue, P. Eades, W. Lai and K. Sugiyama, Layout Adjustment and the Mental Map, J. of Visual Languages and Computing, June 1995, pp. 183-210.

21. A. Papakostas, Information Visualization: Orthogonal Drawings of Graphs, Ph.D. Thesis, University of Texas at Dallas, 1996.

22. A. Papakostas, J. M. Six and I. G. Tollis, Experimental and Theoretical Results in Interactive Orthogonal Graph Drawing, Proc. GD '96, LNCS 1190, SpringerVerlag, 1997, pp. 371-386.

23. A. Papakostas and I. G. Tollis, Algorithms for Area-Efficient Orthogonal Drawings, Computational Geometry: Theory and Applications, 9(1998) 1998, pp. 83-110.

24. A. Papakostas and I. G. Tollis, Issues in Interactive Orthogonal Graph Drawing, Proc. GD '95, LNCS 1027, Springer-Verlag, 1995, pp. 419-430. Also available at http://www .utdallas.edu/ tollis/papers.html.

25. H. Purchase, Which Aesthetic has the Greatest Effect on Human Understanding, Proc. of GD '97, LNCS 1353, Springer-Verlag, 1997, pp. 248-261.

26. M. Schäffter, Drawing Graphs on Rectangular Grids, Discr. Appl. Math., 63(1995), pp. $75-89$.

27. R. Tamassia, On Embedding a Graph in the Grid with the Minimum Number of Bends, SIAM J. Comput., 16(1987), pp. 421-444.

28. R. Tamassia, G. Di Battista and C. Batini, Automatic Graph Drawing and Readability of Diagrams, IEEE Transactions on Systems, Man, and Cybernetics, 18(1), 1988, pp. 61-79.

29. R. Tamassia and I. G. Tollis, Planar Grid Embeddings in Linear Time, IEEE Trans. on Circuits and Systems CAS-36, 1989, pp. 1230-1234.

30. I. G. Tollis, Graph Drawing and Information Visualization, ACM Computing Surveys, 28A(4), December 1996. Also available at http://www.utdallas.edu/ $\sim$ tollis/SDCR96/TollisGeometry/. 